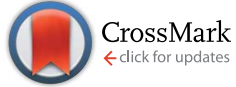

Cite this: J. Mater. Chem. C, 2015, 3, 1235

Received 27th October 2014

Accepted 2nd December 2014

DOI: $10.1039 / c 4 t c 02444 c$

www.rsc.org/MaterialsC

\section{Reversible $\mathrm{J}$ - to $\mathrm{H}$-aggregate transformation in thin films of a perylenebisimide organogelator $\uparrow$}

\author{
Alexandru Sarbu, Laure Biniek, Jean-Michel Guenet, Philippe J. Mésini* \\ and Martin Brinkmann*
}

A perylene bisimide organogelator is shown to behave as a reversible stimuli responsive material: thermal annealing and contact with organic non solvents allow to switch back and forth between a green J-type (Form I) and a red $\mathrm{H}$-type (Form II) aggregate in thin films and powders of a $\mathrm{N}, \mathrm{N}^{\prime}$-substituted $\mathrm{H}$-bonding perylenebisimide (PBI-C10). Both, Form I and II were characterized by transmission electron (low dose high-resolution and electron diffraction) and atomic force microscopies, UV-vis and FTIR spectroscopies. The Form I $\rightarrow$ Form II transformation implies a redistribution of inter-molecular $\mathrm{H}$ bonds between PBI molecules that form columnar stacks in Form I and supramolecular helices with enhanced long-range stacking in Form II. The reverse transformation is triggered by a contact of Form II films with $\mathrm{H}$-bonding organic non solvents e.g. linear alcohols. It is proposed that solvent molecules diffusing in the Form II films can disrupt long-range $\mathrm{H}$-bonding within helical stacks of Form II. Accordingly, PBI-C10 is shown to behave as a functional material responding successively to thermal and molecular stimuli.

\section{Introduction}

Organic dyes whose properties e.g. fluorescence or light absorption change as a response to external stimuli (pressure, light, temperature, presence of organic molecules) are attracting much attention as new smart materials used in optical, electronic or opto-electronic sensors. ${ }^{1}$ Such systems require the possibility to switch between two states corresponding to different optical and/or electronic properties. It is also important that the conversion is reversible. ${ }^{1}$ In this perspective, both absorption and fluorescence of perylene tetracarboxylic acid bisimides (PBIs) systems have been exploited to design stimuliresponsive materials. ${ }^{2}$ PBI-based materials are particularly attractive given their unique electronic properties e.g. n-type charge transport, strong photoluminescence, photoconductivity and photovoltaic activity. ${ }^{3}$ Most importantly, the PBI aromatic core can be substituted in various positions (imide, ortho or bay), making it a unique building block for the design of new electro-active materials such as alternated co-polymers, donor-acceptor co-oligomers, organogelators or stimuliresponsive materials. ${ }^{4}$ For this latter class of materials, Würthner and coworkers demonstrated that complexation of melamine-substituted PBI dyes in solution with cyanurates transforms H- into J-aggregates. ${ }^{5}$ In thin films, Mizoshita and

Institut Charles Sadron, 23 rue du Loess, 67034, Strasbourg, France. E-mail: Philippe. mesini@ics-cnrs.unistra.fr; Martin.brinkmann@ics-cnrs.unistra.fr

$\uparrow$ Electronic supplementary information (ESI) available. See DOI: $10.1039 / \mathrm{c} 4 \mathrm{tc} 02444 \mathrm{c}$ coworkers evidenced mechanochromic properties of a PBI dye bearing siloxane-based flexible substituents: ${ }^{6}$ the change in fluorescence upon application of a mechanical stimulus was related to a change from the amorphous to the crystalline phase. This system is one of the rare examples where the reverse transformation could be realized by a different stimulus as it was induced by solvent vapor exposure. As a general rule, switching of optical properties in the solid state in PBI-based materials implies a structural transformation between different crystalline, liquid-crystalline and/or amorphous states. ${ }^{7}$ It is indeed known that the stacking of PBI dyes leads to a characteristic crystallochromism with either $\mathrm{J}$ - or $\mathrm{H}$-type aggregates whose optical absorption depends closely on the overlap between PBIs in the crystal. ${ }^{8}$ However, although various preparation routes can indeed lead to different polymorphs in PBIs, ${ }^{\mathbf{8}, 9}$ the possibility to switch back and forth between them by two different external stimuli has, to the best of our knowledge, only marginally been addressed so far.

Herein we focus on $N, N^{\prime}$-bis[2-3,4,5-tris $(((S)$-3,7-dimethyloctyl) oxy)benzoylaminoethyl]perylene-3,4,9,10-tetracarboxylic diimide (PBI-C10) which has been originally described by Würthner and coworkers $^{4}$ as leading to J-type fibrillar aggregates upon casting from $p$-xylene. Using UV-vis and FT-IR spectroscopies as well as AFM and TEM, we uncover means to interconvert two polymorphs of this molecule that correspond to $\mathrm{J}$ - and H-type aggregates, highlight their structural characteristics and demonstrate the redistribution of inter-molecular H-bonds upon structural transformation triggered by thermal annealing or exposure to certain organic solvents. 


\section{Experimental section}

$N, N^{\prime}$-bis[2-3,4,5-tris(((S)-3,7-dimethyloctyl)oxy)benzoylaminoethyl]perylene-3,4,9,10-tetracarboxylic diimide (PBI-C10) was synthesized according to the synthetic method described in the ESI (see Fig. S1 $\dagger$ ). The purity of the final product was checked by HPLC chromatography. It was dissolved in a solution of LiBr in NMP $(100 \mathrm{mM})$ and an aliquot was injected on a PL gel column. The eluent was detected by refractometry and in the UV by using a diode array. As an illustration, the trace of the UV detector at $488 \mathrm{~nm}$ is given in Fig. S2. $\dagger$

\section{TEM}

The films were cast on glass slides by using the doctor blade technique starting from a $50 \mu \mathrm{L}$ solution in $p$-xylene $(3 \mathrm{mg}$ $\mathrm{mL}^{-1}$ ). The thin films were detached from the glass slides using $10 \%$ HF solution and were recovered on copper TEM grids. Polarized optical microscopy was performed on a Leica DMR-X microscope equipped with a Nikon Coolpix 995 digital camera. Transmission electron microscopy was performed with a Philips CM $12(120 \mathrm{keV})$ microscope equipped with a CCD MVIII digital camera. The image treatments were carried out using AnalySYS (Soft Imaging Systems) and ITEM (FEI) software. To probe the morphology of the fibrillar aggregates of Form I, $5 \mu \mathrm{L}$ of the solution in $p$-xylene were deposited on a carbon-coated copper grid and subsequently blotted with a filter paper to remove non adsorbed material. For Form II, grids of Form I were subjected to an annealing in a Linkam hot stage (vide infra).

\section{AFM}

For AFM imaging, PBI-C10 solutions were cast on silicon wafers. The observations were made in peak force tapping mode on a Bruker Multimode controlled by a Nanoscope $\mathrm{V}$ module with a $\mathrm{Si}_{3} \mathrm{~N}_{4}$ tip with a constant of $0.4 \mathrm{~N} \mathrm{~m}^{-1}$ and a terminal radius below $5 \mathrm{~nm}$ oscillating at a frequency between 250 and $300 \mathrm{kHz}$. The image treatment was carried out using Nanoscope Analysis software.

\section{UV-vis}

The UV-visible spectroscopy measurements were performed with an Agilent Cary 5000 spectrometer. The in situ temperature annealing experiment were carried out at a rate of $5{ }^{\circ} \mathrm{C} \mathrm{min}^{-1}$ for both heating and cooling ramps using a Linkam LTS 420 heating plate fitted inside the sample chamber of the spectrometer. To study the kinetics for the Form II - Form I transition upon exposure to liquid solvents, the following procedure was used. First, a thin film of Form I PBI-C10 was prepared by drop-casting a solution in $p$-xylene on the inner surface of a Hellma QS 100 cell. After thermal treatment at $190^{\circ} \mathrm{C}$ and slow cooling, a Form II film was obtained. This quartz cell supporting the Form II film was placed in the UV-vis spectrometer and at $t$ $=0 \mathrm{~s}$, it was filled with the solvent and the transformation kinetics was recorded. The concentration of PBI-C10 Form I is calculated by using the intensity of the characteristic $623 \mathrm{~nm}$ peak. This peak is seen as a weak shoulder in pure Form II films corresponding to an intensity $I_{623} \mathrm{~nm}\left(t_{0}\right)$. Furthermore, at the end of the transformation kinetics i.e. when $I_{623} \mathrm{~nm}(t)$ reaches a plateau, $100 \%$ of Form I is obtained (corresponding to an intensity $\left.I_{623} \mathrm{~nm}\left(t_{\mathrm{f}}\right)\right)$. Therefore the relative concentration of Form I generated during solvent exposure at time $t$ is given by:

$$
\begin{gathered}
C_{\text {Form I }}(t)=\frac{I_{623 \mathrm{~nm}}(t)-I_{623 \mathrm{~nm}}\left(t_{0}\right)}{I_{623 \mathrm{~nm}}\left(t_{\mathrm{f}}\right)-I_{623 \mathrm{~nm}}\left(t_{0}\right)} \text { and } \\
C_{\text {Form I }}(t)=1-C_{\text {Form II }}(t) .
\end{gathered}
$$

\section{FTIR}

The FTIR spectra were measured with a Bruker Vertex 70 spectrophotometer in transmission mode. The films of Form I were deposited on a $\mathrm{NaCl}$ plate by the doctor blade technique from a solution in $p$-xylene $\left(5 \mathrm{~g} \mathrm{~L}^{-1}\right)$. The Form II films were obtained by annealing films of Form I on $\mathrm{NaCl}$ in a Linkam heating stage under nitrogen at $190{ }^{\circ} \mathrm{C}$ and slowly cooled down to room temperature $\left(0.5^{\circ} \mathrm{C} \min ^{-1}\right)$.

\section{DSC and TGA}

The DSC measurements were performed with a PerkinElmer DSC 8500. NMR spectra were measured with a Bruker Advance 400 apparatus operating at $400 \mathrm{MHz}$ for ${ }^{1} \mathrm{H}$ and $100 \mathrm{MHz}$ for ${ }^{13} \mathrm{C}$. The TGA measurements were performed with a Mettler TG50 microscale and a Mettler TC10A controller. For these experiments, the powders were placed in alumina crucibles under $\mathrm{N}_{2}$ and temperature was increased at $10{ }^{\circ} \mathrm{C} \mathrm{min}^{-1}$.

\section{Results and discussion}

Herein, we demonstrate that thermal annealing and solvent treatments induce switching back and forth from a J- to a H-type aggregate for a PBI-based organogelator. The organogelator molecule investigated is PBI-C10. It was first described by Würthner and is composed of a PBI core $N, N^{\prime}$ substituted with a flexible ethyl linker, a hydrogen bonding amide and a trialkoxyphenyl dendron (see Fig. 1a). The latter unit bears chiral 2,6-dimethyl-octyl chains. The resulting organogelator molecules form one-dimensional self-assembled fibrils in numerous organic solvents such as $p$-xylene. ${ }^{4}$ When PBI-C10 is cast from a solution in $p$-xylene at $3 \mathrm{~g} \mathrm{~L}^{-1}$, a green film is formed (Fig. $1 \mathrm{~b}$ ). The absorption spectrum in Fig. 1c is characteristic of a J-type aggregate with a strong peak at $625 \mathrm{~nm}$ and a structured band centered around $450 \mathrm{~nm}$, in agreement with previous reports (see ref. 4c). Polarized optical Microscopy (POM) shows almost no birefringence of the cast films. When annealed at $190^{\circ} \mathrm{C}$ i.e. slightly below the melting temperature $\left(T_{\mathrm{m}}=203{ }^{\circ} \mathrm{C}\right.$, see Fig. S3 $\dagger$ ) and slowly cooled to RT, the films display a strong birefringence in POM, indicating a higher level of structuration, not a quenched amorphous phase, and the films turn to red. The UV-vis absorption has changed substantially, the $623 \mathrm{~nm}$ peak has disappeared almost totally and the broad structured band around $450 \mathrm{~nm}$ is replaced by a set of three dominant 


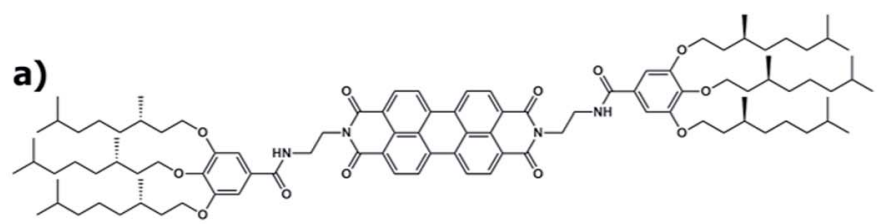

b)

Form I

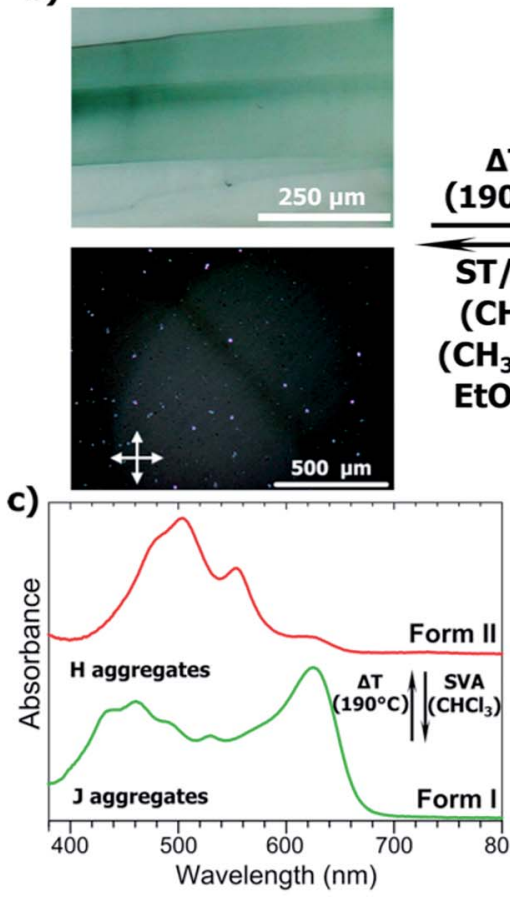

Form II
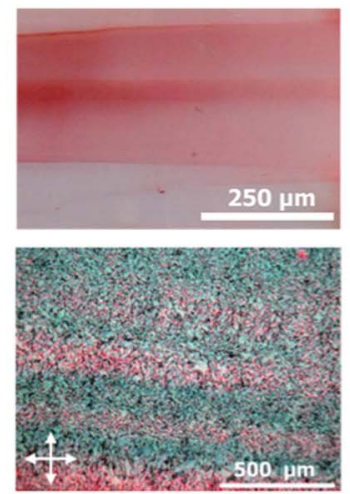

d)

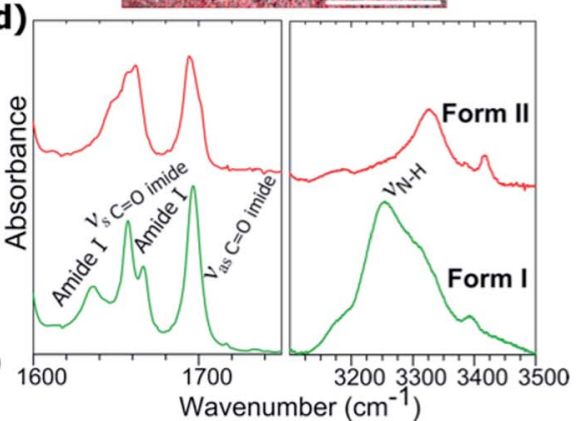

Fig. 1 (a) Molecular structure of the PBI-C10 molecule. (b) Form I $\rightarrow$ Form II transition induced by thermal annealing at $190{ }^{\circ} \mathrm{C}$ and slow cooling to RT. The reverse transition Form II $\rightarrow$ Form I is induced by solvent vapor annealing using $\mathrm{CHCl}_{3}$ vapors (classical solvent vapor treatment, SVA) or solvent treatments (ST) i.e. by dipping the thin film in liquid linear alcohols (ethanol-propanol). Top row: optical microscopy images; bottom row: POM images with crossed polarizers. (c) UV-vis absorption spectra. (d) FTIR spectra.

bands centered at 480,505 and $555 \mathrm{~nm}$. Such a spectrum is characteristic of H-type PBI aggregates, ${ }^{8}$ indicating that thermal annealing at $190{ }^{\circ} \mathrm{C}$ results in a thermally irreversible $\mathrm{J} \rightarrow \mathrm{H}$ aggregate transformation of PBI-C10. The J-type green and $\mathrm{H}^{-}$ type red forms are christened Form I and II, respectively (this transformation is equally observed for powders and films regardless of the substrate used for thin films).

The $\mathrm{H}$ to $\mathrm{J}$ transition observed originates from a change in the packing of the aromatic cores. We surmise that this reorganization also involves part of the compounds and different intermolecular interactions. The hydrogen-bonds that involve the amide groups between successive PBI-C10 molecules were explored by FT-IR spectroscopy (see Fig. 1d). The amide A band corresponding to the stretching of the $\mathrm{N}-\mathrm{H}$ bond is particularly sensitive to $\mathrm{H}$-bonding interactions and their intensities. ${ }^{10}$ As seen in Fig. 1d, the FT-IR spectra of both forms are indeed very different. In Form I, the two bands at $3310 \mathrm{~cm}^{-1}$ and $3250 \mathrm{~cm}^{-1}$ correspond to strongly $\mathrm{H}$-bonded and moderately $\mathrm{H}$-bonded amides, respectively (other weaker bands can be observed at 2075, 3181 and $3392 \mathrm{~cm}^{-1}$. From the literature and by comparison with model compounds, they have been assigned respectively as amide $\mathrm{A}^{\prime}$, harmonic of aromatic $\mathrm{C}=\mathrm{C}$ str. and harmonic of asymmetric imide CO str.). Form II is characterized by two major bands centered at $3328 \mathrm{~cm}^{-1}$ and $3426 \mathrm{~cm}^{-1}$. The first band corresponds to amides with moderate H-bonds. The second band has a much lower width and is characteristic of non-bonded amides. The CO stretching area confirms these facts: Form I exhibits two amide I bands at 1636 and $1665 \mathrm{~cm}^{-1}$, that correspond to strongly and moderately bonded amides, respectively. In the Form II the peak at $1665 \mathrm{~cm}^{-1}$ disappears while a shoulder appears at $1680 \mathrm{~cm}^{-1}$ characteristic of free amides. In parallel, UV-vis and FT-IR spectroscopies indicate a strong re-organization of the molecular packing within the PBIC10 films upon annealing, transforming initial J-type to H-type aggregates. Surprisingly however, the Form II with the highest order (both in POM and electron diffraction, vide infra), shows non-bonded amides whereas the Form I grown from solution shows the strongest $\mathrm{H}$-bonds between amides. The presence of non-bonded amides in Form II can tentatively be attributed to a non-symmetric $\mathrm{H}$-bonding between successive PBI-C10 molecules in columnar stacks, preventing some amides to find a neighboring molecule for $\mathrm{H}$-bonding. 
Except for a prominent melting endotherm $\left(T_{\mathrm{m}}=203{ }^{\circ} \mathrm{C}\right)$, DSC of Form I powders (Fig. S3†) does not reveal a well defined structural transition but only a small peak $\left(\Delta H=1.6 \mathrm{~kJ} \mathrm{~mol}^{-1}\right)$ whose position shifts to higher temperatures with increasing heating rate between $185{ }^{\circ} \mathrm{C}$ and $189{ }^{\circ} \mathrm{C}$ when the rate changes from 5 to $15{ }^{\circ} \mathrm{C} \mathrm{min}^{-1}$. This peak is not observed during the second heating cycle, i.e. once Form I has been converted to Form II, which suggests that it is related to the Form I $\rightarrow$ Form II transformation and ultimately that the prominent melting endotherm corresponds to the melting of Form II.

Further evidence for the structural reorganization were obtained by both AFM and TEM (Fig. 2). As shown by AFM and bright field TEM, PBI-C10 in both Form I and II generates fibrillar aggregates several microns long and a few tens of nanometers wide, with an average width of the fibrils slightly larger in Form II. Section profiles of the AFM topographic images show the existence of terraces atop the fibrillar structures. The measured heights of the terraces that are statistically recurrent are, $27 \AA$ and $33 \AA$ for Form I and 52, 31 and $27 \AA$ for Form II. The structures differ significantly as indicated by low dose High Resolution TEM (HR-TEM) and electron diffraction (ED) (see Fig. 2 and 3). HRTEM images in Fig. 2d and h reveal columnar stacks of Form I and II with inter-columnar distances of $33 \AA$ and $26 \AA$, respectively (the contrast in the HR-TEM image is related to the alternation of columns of PBI cores and layers of disordered alkyl side chains of the trialkoxyphenyl dendrons). The ED of Form I displays a reflection at $d_{h k l}=34 \pm 1 \AA$ corresponding to the inter-columnar period and a broad and weak Scherrer ring corresponding to an inter-molecular distance of $4.8 \AA$ within stacks (Fig. 3a). As seen in Fig. 3b, the ED pattern of Form II shows a much larger set of reflections at 26.2 ̊, 17.5 ̊, $13.0 \AA$ А, $10.6 \AA, 8.9 \AA, 7.6 \AA$ and $4.6 \AA$. The $26 \AA$ peak corresponds to the inter-column period and the $4.6 \AA$ peak to the molecular stacking period within columns. These ED patterns indicate that the structures of Form I and II are significantly different: both involve some "columnar" stacks but with different inter- and intra-column stacking periodicities. The observed intra-molecular stacking period is close to those observed in hydrogen-bonded PBIs. ${ }^{11}$ This distance is closer to the amide-amide distance in H-bonded systems (typically $4.9 \AA$ in nylon-6) than the $3.6 \AA \pi$-stacking characteristic of PBIs. ${ }^{8}$ This suggests that the stacking of PBI-C10 in both polymorphs is enforced by $\mathrm{H}$ bonding rather than $\pi$-stacking and that the PBI cores are possibly tilted within stacks to allow for shorter $\pi$ $\pi$ stacking distances. ${ }^{11}$

The close analysis of the ED pattern of Form II indicates further substantial differences with respect to Form I. The Fourier transform of the HR-TEM image and the ED of Form II show a set of strong reflections characterized by a long period, $P$, of $97 \AA$ along the stacking direction (see Fig. 3). The coexistence of both, a short inter-molecular stacking period, $p=4.6 \AA$ and a long period, $P=97 \AA$ is supportive of a helical stacking of PBI-C10 molecules in Form II. ${ }^{12}$ The helical period corresponds to 20-21 molecular units stacked at an intermolecular distance of 4.6 ̊. The packing of PBI-based molecules in supramolecular helices has been reported previously by Percec et al. ${ }^{13}$ However, in the latter study, the PBI systems did not involve a H-bonding amide but a flexible polymethylene linker between the PBI core and the two trialkyloxy-benzene dendrons. The presence of inter-molecular H-bonds in PBI-C10 may account for the large helical period of $97 \AA$ observed in Form II as compared to the 14-15 A period observed in the systems investigated previously by Percec et al. ${ }^{13}$

The transformation Form I $\rightarrow$ Form II was followed by UVvis absorption (Fig. 4a). The spectrum of Form I shows a progressive change with increasing temperature: the $623 \mathrm{~nm}$ peak both decreases continuously in intensity and shifts to lower wavelength in the range $25{ }^{\circ} \mathrm{C} \leq T \leq 150{ }^{\circ} \mathrm{C}$. The absence of isosbestic points suggests that the Form I $\rightarrow$ Form II transformation could imply intermediate states between Form I and II. This in turn could explain the absence of a sharp and well defined transition in the DSC (see Fig. S3 $\dagger$ ).

In order to consider $\mathrm{PBI}-\mathrm{C} 10$ as a functional material responsive to a thermal stimulus, it is necessary to find a mean

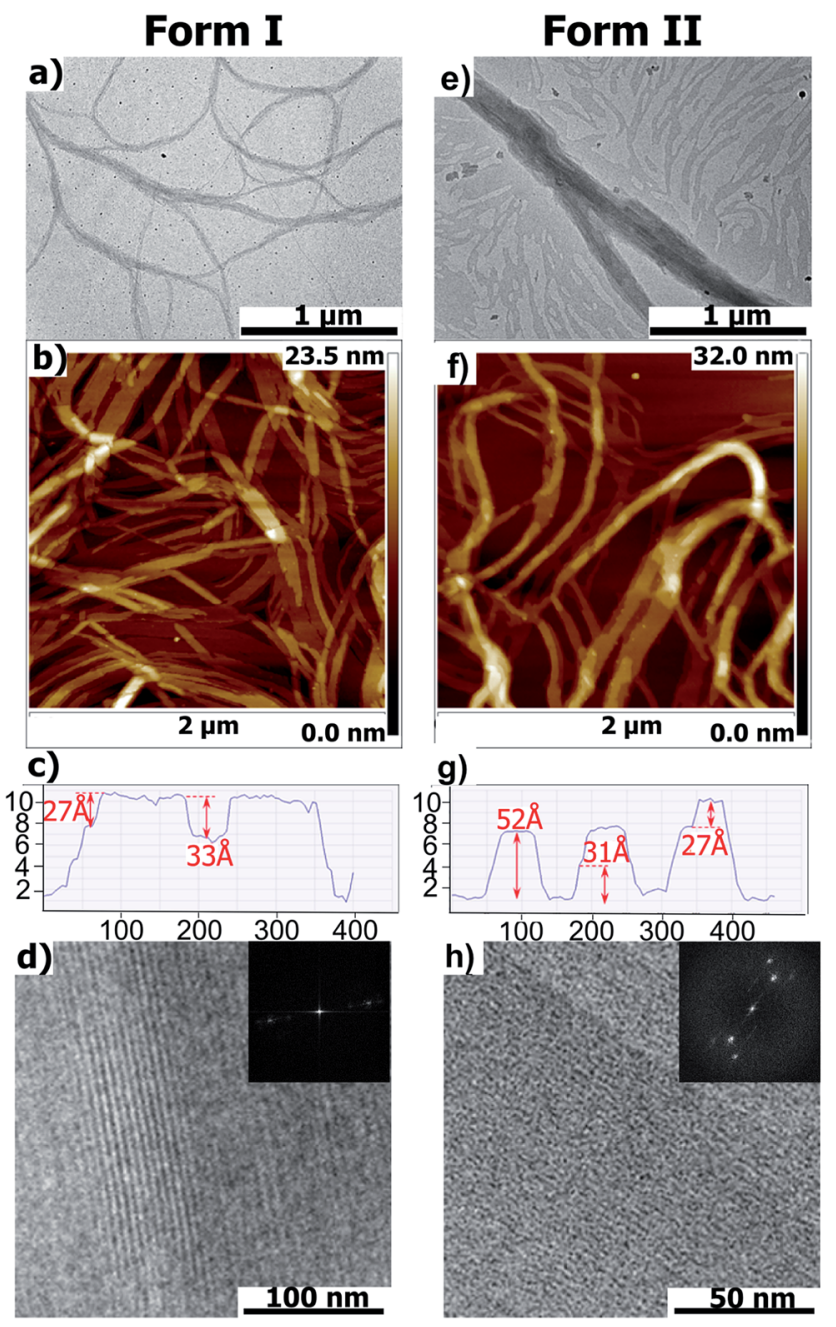

Fig. 2 Morphology of PBI-C10 in Form I and II. (a) \& (e) TEM bright field images; (b) \& (f) AFM images; (c) \& (g) section profile of an AFM topographic image showing the presence of characteristic terraces; (d), (h) low dose HR-TEM images (inset: fast Fourier transform). In Form II, note the presence of some intense reflections in the first layer line above the equator which is a fingerprint of a helical molecular packing. 

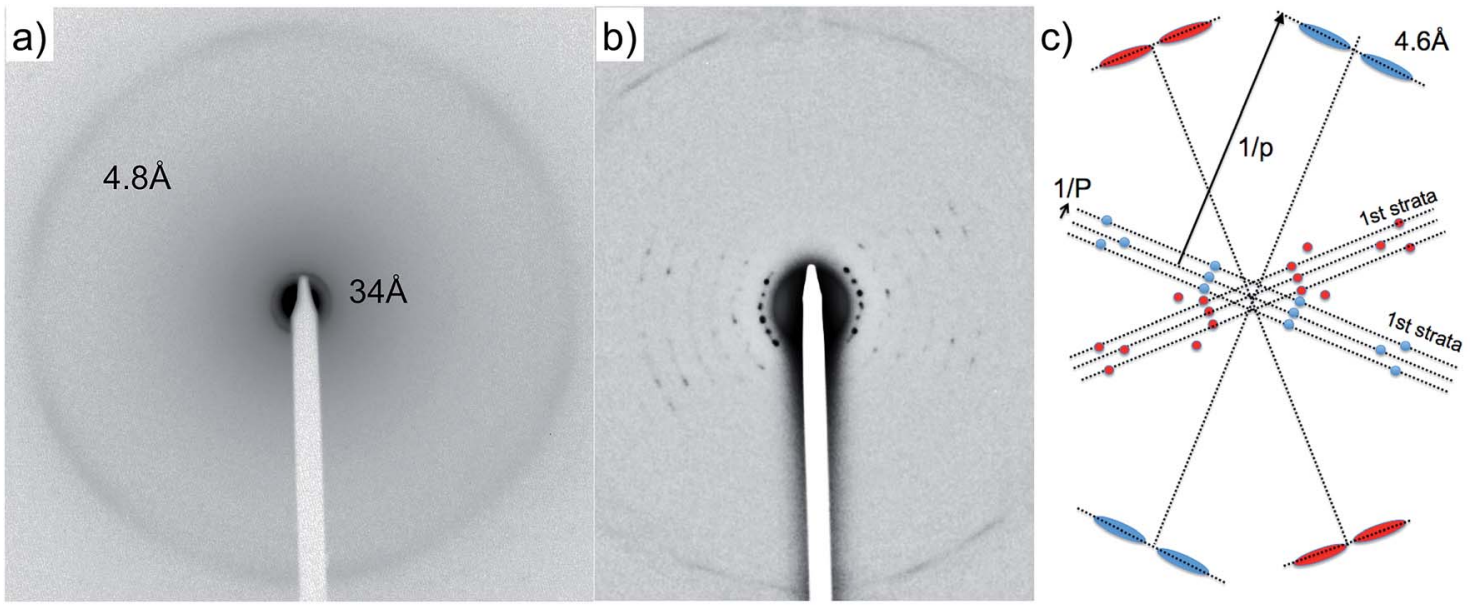

d)
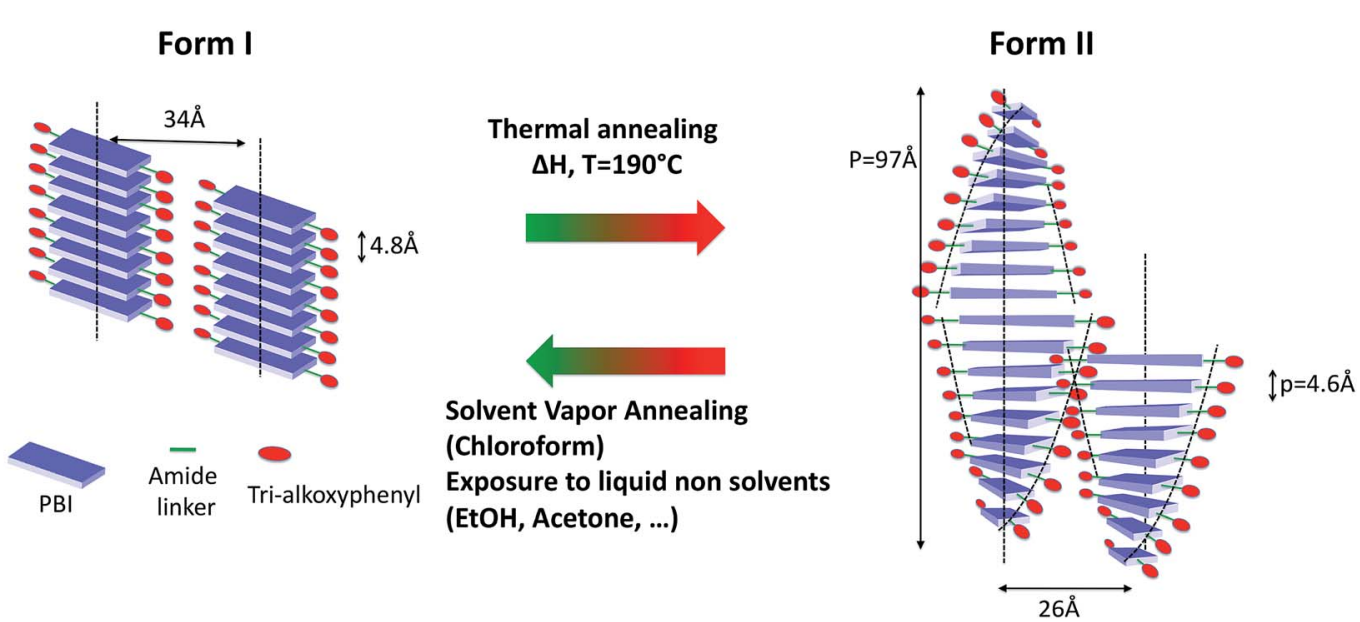

Fig. 3 Experimental ED pattern of Form I (a) and Form II (b) of PBI-C10. (c) Schematic interpretation of the composite ED pattern of Form II. The pattern is the overlap of two single crystal-like patterns depicted in red and blue. Both these patterns show a broad and diffuse slightly offmeridional reflection corresponding to a stacking period $p=4.6 \AA$ as well as two intense and off-meridional reflections on a first strata corresponding to a large period $P=97 \AA$. The ratio $P / p$ suggests that $20-21$ molecules are stacked in a helix with a period of $97 \AA .{ }^{12}$ (d) Schematic illustration of the structures of the green Form I and red Form II of PBI-C10 and the reversible transformation methods. Form I and II involve columnar and helical stacks, respectively.

to recover the original Form I. Solvent vapor annealing is a common method to induce structural changes in thin films of conjugated dyes in general and molecular semiconductors in particular. ${ }^{14}$ Exposure of Form II films of PBI-C10 to vapors of a good solvent such as $\mathrm{CHCl}_{3}$ (solvent vapor annealing) does indeed induce a transformation back to the original green Form I as illustrated in Fig. 1a. This transformation results from a simple dissolution of Form II in $\mathrm{CHCl}_{3}$ and re-crystallization to Form I, underlining the important role of the solvent used for the Form I preparation. More interestingly, the Form II $\rightarrow$ Form I transformation is also induced by bringing Form II films in contact with liquid non solvents such as acetone or linear alcohols (from 1-methanol to 1-butanol). ${ }^{\mathbf{1 5}}$ However, not all organic non-solvents induce such a transformation. Form II films are unaltered by alkanes or aromatic solvents (benzene, toluene). The solvents must bear a hydrogen bonding group to induce the structural transformation of PBI-C10.
The Form II $\rightarrow$ Form I transformation by contact with linear alcohols and its kinetics were followed by UV-vis absorption spectroscopy (see Fig. 4b). As seen in Fig. 4b, two isosbestic points are observed, underlining the direct transformation of Form II to Form I without intermediate species. The kinetics is of first order i.e. the concentration of Form II varies according to $C_{\mathrm{II}}(t)=C_{\mathrm{II}}^{0} \mathrm{e}^{-k t}$ where $k$ is the rate constant of the reaction and $C_{\mathrm{II}}^{0}$ is the initial concentration of Form II. $k$ increases with the alcohol length, typically $k=0.185 \mathrm{~min}^{-1}$ for butanol and 0.013 $\min ^{-1}$ for ethanol (see inset in Fig. 4c). ATG experiments (see Fig. $\mathrm{S} 4 \dagger$ ) do not evidence any retention of non-solvent by Form II powders, indicating that a massive bulk diffusion of those linear alcohol molecules in Form II is not at the origin of the phase transformation (moreover, its kinetics would be diffusion-limited and follow a Fickian time dependence).

Non solvents such as alcohols (ethanol or $n$-butanol) as well as acetone are known to induce polymorphic modifications in $\pi$-conjugated semiconductors such as lithium phthalocyanine 
a)

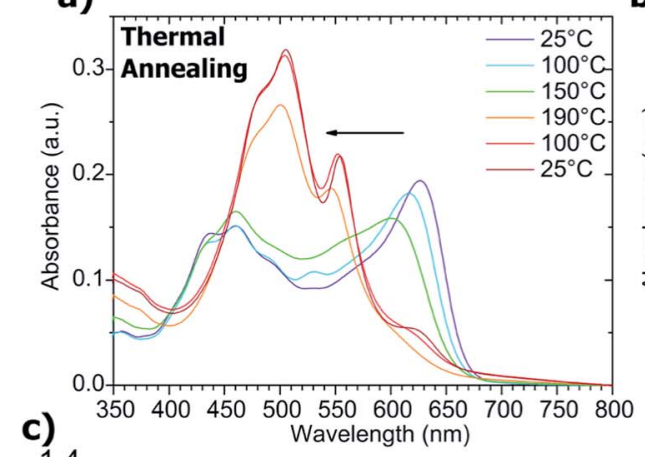

b) 1
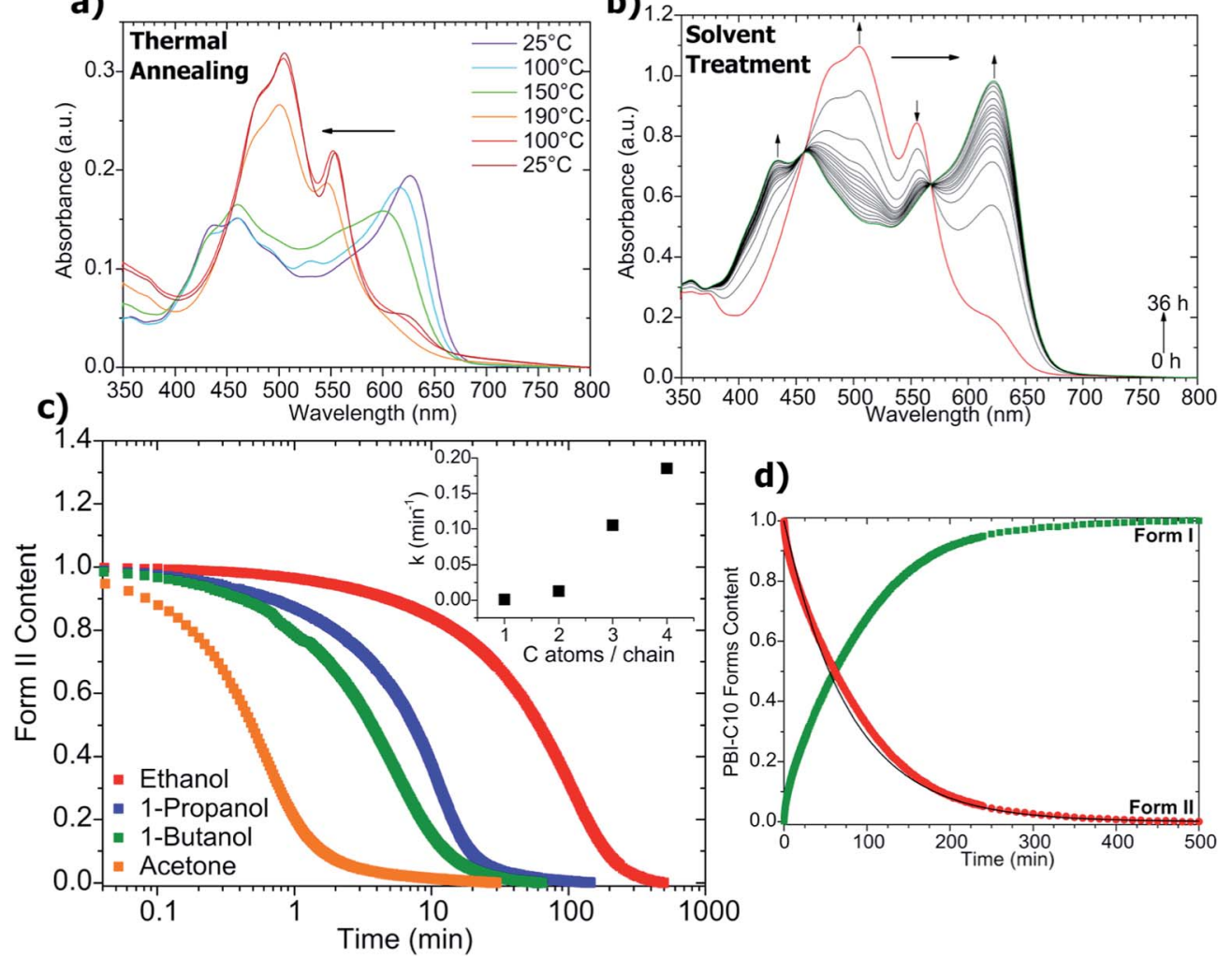

d)

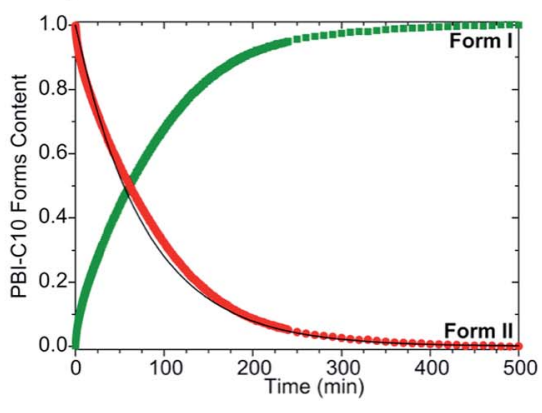

Fig. 4 (a) Evolution of the UV-vis absorption spectrum of a PBI-C10 Form I thin film heated up to $210^{\circ} \mathrm{C}$ and cooled to $25^{\circ} \mathrm{C}$. (b) Evolution of the UV-vis absorption spectrum of a PBI-C10 Form II thin film placed in contact with ethanol. (c) Evolution of the Form II concentration in a PBI-C10 Form II thin films placed in various "non-solvents"; inset: variation of the rate constant $k$ on the number of carbon atoms in the alcohol chain. (d) Time-evolution of the Form I and II concentration in a PBI-C10 Form II thin film placed in ethanol. The fit corresponds to a first order kinetics (see text).

(LiPc) and zinc phthalocyanine (ZnPc).$^{14} \mathrm{In} \mathrm{ZnPc}$, a weak charge transfer occurs between the lone electronic pairs of the solvent and the $\mathrm{ZnPc}$ molecules located at the surface of the initial $\alpha$ form crystals. The resulting variation in the electronic densities of ZnPc triggers the change in molecular packing from the $\alpha$ to the $\beta$ phase.$^{14}$ While a similar mechanism might occur for PBI$\mathrm{C} 10$, one must also take into account the presence of $\mathrm{H}$-bonding in PBI-C10. Contrary to phthalocyanines whose packing is essentially governed by van der Waals interactions, the structural modifications in PBI-C10 involve additional $\mathrm{H}$ bonds that are responsible for the generation of long-range $\mathrm{H}$-bonded helical stacks in Form II. As demonstrated by FTIR, a redistribution of H-bonds is involved in the Form I $\rightarrow$ Form II transformation with the presence of a characteristic population of non bonded amides in Form II. A modification of $\mathrm{H}$-bonding is also involved in the reverse transformation when Form II converts to Form I in the presence of H-bonding non solvents. A possible mechanism for this structural transformation could imply some $\mathrm{H}$-bonding between the PBI-C10 and the solvent molecules diffusing inside the Form II films. This situation reminds the case of solvent induced crystallization of some poly(amides) such as nylon $6 \mathrm{I} .{ }^{16}$ Crystallization of nylon $6 \mathrm{I}$ is induced by contacting the polymer with hydrogen bond-forming non solvents such as linear alcohols. In the case of PBI-C10,
H-bonding non solvent molecules could break the long-range helical stacks of Form II into smaller multimolecular aggregates. PBI tetramers that organize into columnar stacks were indeed evidenced in the ordered phases of PBI systems studied by Percec et al. ${ }^{13}$ This interpretation would further agree with the observation of limited/poor stacking order in Form I (ED and POM) as compared to Form II despite fairly similar morphologies of the fibrillar objects.

\section{Conclusion}

Two polymorphs of a hydrogen bonding PBI organogelator with typical J-and H-type aggregate properties can be interconverted using two different stimuli i.e. temperature and presence of $\mathrm{H}^{-}$ bonding non solvents. Contrary to earlier reports showing that peripheral substituents of PBI induce either H-type or J-type aggregates, it is demonstrated that the same molecular system can exhibit both type of aggregates in thin films provided the right preparation protocol is followed. The green J-type Form I transforms into the red H-type Form II via annealing at $190{ }^{\circ} \mathrm{C}$ whereas the reverse transformation is induced when the Form II films are soaked in liquid H-bonding non-solvents such as linear alcohols or acetone (see Fig. 3). This structural transformation rests on a redistribution of H-bonds as inferred by 
the analysis of the amide A bands as well as structural studies by AFM and TEM. Interestingly, Form II PBI-C10 molecules generate helical stacks with a long period of $97 \AA$ A. Contrary to Form I, Form II contains a population of non-bonded amides, possibly related to a non-symmetric H-bonding between successive PBI molecules within helical stacks. Incidentally, the peculiar polymorphism of the PBI-C10 organogelator illustrates how long range supramolecular assemblies formed by PBI derivatives can be used as stimuli-responsive materials that can respond reversibly to temperature and exposure to $\mathrm{H}$-bonding non-solvents such as alcohols. It can be anticipated that a finetuning of the stimulus response might be achieved by proper choice of H-bonding dendrons and by controlling the length of the amide linker between the PBI core and the trialkoxyphenyl dendrons.

\section{Acknowledgements}

This work is supported by a Région Alsace fellowship (A.S.) and by the Agence Nationale pour la Recherche (project ANR-11BS08-0001). Bernard Lotz is gratefully acknowledged for fruitful discussions and reading of the manuscript. Cathy Saettel is acknowledged for performing the DSC experiments. Christophe Contal, Christian Blanck and Fabienne Hubert are acknowledged for technical support in AFM, TEM and UV-vis spectroscopy, respectively.

\section{References}

1 (a) Y. Shiki, in Supramolecular soft matter, ed. N. Takashi, Springer, 2011, pp. 77-95; (b) T. Ishi-i and S. Shinkai, Top. Curr. Chem., 2005, 258, 119; (c) Y. Sagara and T. Kato, Nat. Chem., 2009, 1, 605; (d) Y. Che, X. Yang, S. Loser and L. Zang, Nano Lett., 2008, 8, 2219; (e) Y. Che, X. Yang, G. Liu, C. Yu, H. Ji, J. Zuo, J. Zhao and L. Zang, J. Am. Chem. Soc., 2010, 132, 5743.

2 F. Würthner, C. Thalacker, S. Diele and C. Tschierske, Chem.-Eur. J., 2001, 7, 2245-2253.

3 (a) A. S. Molinari, H. Alves, Z. Chen, A. Facchetti and A. F. Morpurgo, J. Am. Chem. Soc., 2009, 131, 2462-2463; (b) H. Yan, Z. Chen, Y. Zheng, C. Newman, J. R. Quinn, F. Dötz, M. Kastler and A. Facchetti, Nature, 2009, 457, 679-686; (c) R. Steyrleuthner, M. Schubert, F. Jaiser, J. C. Blakesley, Z. Chen, A. Facchetti and D. Neher, Adv. Mater., 2010, 22, 2799-2803; (d) M. Schubert, D. Dolfen, J. Frisch, S. Roland, R. Steyrleuthner, B. Stiller, Z. Chen, U. Scherf, N. Koch, A. Facchetti and D. Neher, Adv. Energy Mater., 2012, 2, 369-380; (e) H. Yu, Z. Bao and J. H. Oh, Adv. Funct. Mater., 2012, 23, 525-536; (f) V. Kamm, G. Battafliarin, I. A. Howard, W. Pisula, A. Mavrinskiy, C. Li, K. Müllen and F. Laquai, Adv. Energy Mater., 2011, 1, 297-302; $(g)$ L. Schmidt-Mende, A. Fechtenkötter, K. Müllen, E. Moons, R. H. Friend and J. D. MacKenzie, Science, 2001, 293, 1119; (h) F. Würthner, Chem. Commun., 2004, 1564-1579; ( $i$ ) F. Würthner, T. E. Kaiser and C. R. Saha-Möller, Angew. Chem., Int. Ed., 2011, 50, 3376; (j) X. Zhan, A. Facchetti, S. Barlow, T. J. Marks, M. A. Ratner,
M. R. Wasielewski and S. R. Marder, Adv. Mater., 2011, 23, 268-284; (k) C. Li and H. Wonneberger, Adv. Mater., 2012, 24, 613-636; (l) E. Kozma and D. Catellani, Dyes Pigm., 2013, 98, 160-179.

4 (a) K. Balakrishnan, A. Datar, T. Naddo, J. Huang, R. Oitker, M. Yen, J. Zhao and L. Zang, J. Am. Chem. Soc., 2006, 128, 7390-7398; (b) B. Jancy and S. K. Asha, Chem. Mater., 2008, 20, 169-181; (c) S. Ghosh, X.-Q. Li, V. Stepanenko and F. Würthner, Chem.-Eur. J., 2008, 14, 11343-11357; (d) Z. Chen, B. Fimmel and F. Würthner, Org. Biomol. Chem., 2012, 10, 5845-5855; (e) L. Bu, X. Guo, B. Yu, Y. Qu, Z. Xie, D. Yan, Y. Geng and F. Wang, J. Am. Chem. Soc., 2009, 131, 13242-13243; (f) P.-O. Schwartz, E. Zaborova, L. Biniek, B. Heinrich, M. Brinkmann, N. Leclerc and S. Mery, J. Am. Chem. Soc., 2014, 136, 5981-5992; $(g)$ N. Mizoshita, T. Tani and S. Inagaki, Adv. Mater., 2012, 24, 3350.

5 S. Yagai, T. Seki, T. Karatsu, A. Kitamura and F. Würthner, Angew. Chem., Int. Ed., 2008, 47, 3367.

6 N. Mizoshita, T. Tani and S. Inagaki, Adv. Mater., 2012, 24, 3350.

7 (a) Y. Sagara, T. Mutai, I. Yoshikawa and K. Araki, J. Am. Chem. Soc., 2007, 129, 1520; (b) Y. Sagara and T. Kato, Angew. Chem., Int. Ed., 2008, 47, 5175.

8 (a) E. Hädicke and F. Graser, Acta Crystallogr., Sect. C: Cryst. Struct. Commun., 1986, 42, 189-195; (b) E. Hädicke and F. Graser, Acta Crystallogr., Sect. C: Cryst. Struct. Commun., 1986, 42, 189-195; (c) G. Klebe, F. Graser, E. Hädicke and J. Berndt, Acta Crystallogr., Sect. B: Struct. Sci., 1989, 45, 6977.

9 (a) R. A. Cormier and B. A. Gregg, J. Phys. Chem. B, 1997, 101, 11004-11006; (b) S. G. Liu, G. Sui, R. A. Cormier, R. M. Leblanc and B. A. Gregg, J. Phys. Chem. B, 2002, 106, 1307; (c) B. A. Gregg, J. Phys. Chem., 1996, 100, 852-859.

10 (a) D. Lin-Vien, N. B. Colthup, W. G. Fateley and W. G. Grasselli, The Handbook of Infrared and Raman Characteristic Frequencies of Organic Molecules, Academic Press, San Diego, 1991, pp. 155-178; (b) N. Diaz, F. X. Simon, M. Schmutz, M. Rawiso, G. Decher, J. Jestin and P. J. Mésini, Angew. Chem., Int. Ed., 2005, 44, 3260-3264.

11 R. Marty, R. Szilluweit, A. Sánchez-Ferrer, S. Bolisetty, J. Adamcik, R. Mezzenga, E.-C. Spitzner, M. Feifer, S. N. Steinmann, C. Corminboeuf and H. Frauenrath, ACS Nano, 2013, 7, 8498-8508.

12 (a) F. H. C. Crick and A. Rich, Nature, 1955, 176, 780; (b) W. Cochran, F. H. Crick and V. Vand, Acta Crystallogr., 1952, 5, 581; (c) R. E. Dickerson and I. Geis, The structure and action of proteins, Harper and Row, New York, 1969.

13 (a) V. Percec, M. Peterca, T. Tadjiev, X. Zeng, G. Ungar, P. Leowanawat, E. Aqad, M. R. Imam, B. M. Rosen, U. Akbey, R. Graf, S. Sekharan, D. Sebastiani, H. W. Spiess, P. A. Heiney and S. D. Hudson, J. Am. Chem. Soc., 2011, 133, 12197-12219; (b) V. Percec, H.-J. Sun, P. Leowanawat, P. Peterca, R. Graf, H. W. Spiess, X. Zeng, G. Ungar and P. A. Heiney, J. Am. Chem. Soc., 2013, 135, 4129-4148.

14 (a) T. Kobayashi, N. Uyeda and E. Suito, J. Phys. Chem., 1967, 72, 2446; (b) F. Iwatsu, T. Kobayashi and N. Uyeda, J. Phys. Chem., 1980, 84, 3223; (c) M. Brinkmann, J.-C. Wittmann, 
C. Chaumont and J.-J. André, Thin Solid Films, 1997, 292, 15 Neither acetone nor the linear alcohols can solubilize PBI192; (d) L. Giovanna, A. Liscio, P. Maccagnani, F. Nolde, C10 (not even at their boiling points).

V. Paleromo, K. Müllen and P. Samori, Soft Matter, 2008, 4, 16 W. Liu, B. Breault and J. Brisson, J. Polym. Sci., Part B: Polym. 2064-2070; (e) L. Giovanna, A. Liscio, F. Nolde, F. Scolaro, Phys., 1995, 33, 619.

L. Monsu, V. Paleromo, K. Müllen and P. Samori, Adv. Funct. Mater., 2007, 17, 3791-3798. 